La variación terminológica en textos fuente y sus traducciones: un estudio piloto

\title{
LA VARIACIÓN TERMINOLÓGICA EN TEXTOS FUENTE Y SUS TRADUCCIONES: UN ESTUDIO PILOTO $^{1}$
}

\author{
Sabela Fernández-Silva \\ Pontificia Universidad Católica de Valparaíso (Chile) / Universidad Pompeu Fabra (España) \\ sabela.fernandez@pucv.cl \\ Koen Kerremans \\ Colegio Erasmo de Bruselas / Universidad Libre de Bruselas (Bélgica) \\ koen.kerremans@vub.ac.be
}

Recibido: 22/04/2020 - Aprobado: 24/04/2020 - Publicada: 15/04/2021

DOI: doi.org/10.17533/udea.lyl.n79a29

Artículo traducido del inglés por Juan Felipe Zuluaga Molina² y Manuela Hernández Sánchez ${ }^{3}$

Resumen: En este artículo, se parte de la hipótesis de que la elección de variantes terminológicas en textos fuente especializados a veces tiene una motivación cognitiva y que esta motivación se refleja en la elección de equivalentes en los textos meta. A partir de un estudio piloto, presentaremos una metodología para comparar las variantes terminológicas motivadas cognitivamente en los textos fuente y sus traducciones. El corpus de este estudio piloto se compone de tres textos fuente en gallego y sus traducciones al inglés; estos textos son artículos científicos que abordan los efectos económicos de los desastres ambientales en la pesca. Primero se realizó un estudio cuantitativo en el que se comparó el número de términos únicos en cada texto fuente con el número de traducciones únicas de estos términos. Luego, cada combinación única de un término fuente y su equivalente se sometió a un análisis cualitativo. Se asignó de forma manual un valor con el fin de calificar la «distancia cognitiva» entre el término fuente y su traducción. Por último, con base en estos valores y la frecuencia del par de traducción en cada texto paralelo, calculamos el «índice de variación interlingüística» (IVI). Las diferencias en los resultados entre los textos paralelos están vinculadas con factores extralingüísticos relacionados con los procesos de traducción.

Palabras clave: variación terminológica; traducción especializada; motivación terminológica; índice de variación interlingüística; distancia cognitiva.

$\overline{1}$ El presente artículo apareció originalmente en la revista canadiense Meta [56(2), 318-335] en el año 2011 bajo el nombre Terminological Variation in Source Texts and Translations: A Pilot Study. El interés por la traducción de este artículo, cuya autorización fue dada por los autores, la revista antes mencionada y la editorial Les Presses de l’Université de Montréal, surgió en el desarrollo del proyecto de investigación Variación terminológica vertical y horizontal en español: hacia una descripción lingüistica de las unidades terminológicas desde la lingüística de corpus (VaTeHoVe), del grupo de investigación en Traducción y Nuevas Tecnologías (TNT), el cual fue aprobado para su financiación por medio del Acta del CODI (Comité para el Desarrollo de la Investigación) de la Universidad de Antioquia n. ${ }^{\circ} 2018-22970$ del 11 de julio de 2019.

2 Miembro del grupo de investigación TNT (Traducción y Nuevas Tecnologías) Escuela de Idiomas, Universidad de Antioquia (Colombia).Correo electrónico:juanf.zuluaga@udea.edu.co

3 Miembro del grupo de investigación TNT (Traducción y Nuevas Tecnologías) Escuela de Idiomas, Universidad de Antioquia (Colombia).Correo electrónico: manuela.hernandezs@udea.edu.co

Editores 
Abstract: In this article, it is assumed that the choice of terminological variants in specialized source texts is sometimes cognitively motivated and that this motivation is reflected in the choice of equivalents in the target texts. On the basis of a pilot study, we will present a method for comparing the cognitively motivated terminological variants in source texts and their translations. The corpus in the pilot study is composed of three Galician source texts and their English translations. The texts are scientific articles addressing the economic effects of environmental disasters on fisheries. A quantitative study was first carried out in which the number of unique terms in each source text was compared to the number of unique translations of these terms. Next, each unique combination of a source term and its translation equivalent was subjected to a qualitative analysis. A value was manually assigned in order to qualify the «cognitive distance» between the source term and its translation. Based on these values and the frequency of the translation pair in each bitext, we computed the «interlingual variation index» (IVI). Differences in results between the bitexts are linked to extra-linguistic factors related to the translation processes.

Key words: terminological variation; specialized translation; term motivation; interlingual variation index; cognitive distance.

Résumé : Dans le présent article, nous soutenons l'hypothèse selon laquelle le choix des variantes terminologiques dans les textes sources est parfois motivé sur le plan cognitif et que cette motivation est reproduite dans le choix des équivalents dans les textes cibles. À partir d'une étude pilote, nous présentons une méthode pour comparer les variantes terminologiques cognitivement motivées dans les textes sources et leurs traductions. Le corpus est constitué de trois articles scientifiques portant sur les conséquences économiques des catastrophes naturelles sur la pêche, écrits originairement en galicien et traduits ensuite en anglais. Nous avons d'abord comparé quantitativement le nombre de termes uniques dans chaque texte avec le nombre de traductions uniques de ces termes. Nous avons ensuite entrepris une analyse qualitative de chaque paire terme source-terme équivalent, dans laquelle nous avons caractérisé la distance cognitive entre ces deux termes à l'aide d'une valeur numérique. À partir de ces valeurs et de la fréquence des paires de traduction dans chaque bitexte, nous avons calculé l'index de variation interlangue (IVI) de chaque texte. Finalement, nous avons mis en relation les différents résultats entre les bitextes avec des facteurs extralinguistiques concernant le processus de traduction.

Mots-clés : variation terminologique ; traduction spécialisée ; motivation terminologique ; index de variation interlangue ; distance cognitive. 
La variación terminológica en textos fuente y sus traducciones: un estudio piloto

\section{Introducción}

T os recientes enfoques descriptivos de la terminología comparten la afirmación de que los términos están sujetos a los mismos dos patrones de variación que las palabras del discurso general: la variación que afecta la forma o variación denominativa y la variación que afecta el contenido o variación conceptual. En este estudio, nuestro interés se centra en los diversos términos que se utilizan para referirse a un único objeto, ya sea dentro de la misma lengua (sinonimia) o entre lenguas diferentes (equivalentes de traducción). A lo largo de este artículo, se utilizará el término «variación terminológica» en lugar de variación denominativa con el fin de enfatizar el hecho de que el estudio se centra solo en la terminología como un medio para expresar un pensamiento específico. Además, en este estudio, no se consideran otros medios de expresión.

Los estudios basados en corpus han demostrado que la variación terminológica es prominente en la comunicación especializada (Freixa, 2002). En la literatura se exponen varias razones para explicar por qué ocurre la variación terminológica (Freixa, 2006). Un autor de un texto especializado puede decidir usar un conjunto de expresiones alternativas por motivos estilísticos para evitar que un pensamiento particular siempre se exprese con exactitud de la misma manera, lo cual no es necesariamente «un acto aleatorio de rebeldía o descuido, sino uno que tiene una motivación explícita y es útil en el discurso especializado» (Bowker, 1998, p. 487). Las formas de expresar un pensamiento también diferirán cuando, por ejemplo, un especialista esté hablando sobre un tema de su área de estudio con un colega en comparación con un no experto. En cada nueva situación comunicativa, un emisor tiene que encontrar qué formas de expresión transmiten mejor un mensaje al receptor con la mayor claridad posible (Cabré, 1995). Los términos establecidos pueden no parecer siempre la mejor opción, ya sea porque el pensamiento que se desea expresar es un poco diferente o porque los términos que se suelen usar para expresar el pensamiento no se ajustan a la forma en la que el emisor lo comprende (Bowker, 1997).

En este artículo, se parte de la premisa de que la elección de variantes terminológicas en textos fuente especializados a veces tiene una motivación cognitiva y que esta motivación se refleja en la elección de equivalentes en los textos meta (Suárez de la Torre, 2004). A partir de un estudio piloto, presentaremos una metodología para comparar las variantes terminológicas motivadas cognitivamente en los textos fuente y sus traducciones. La metodología se deriva de dos proyectos de doctorado sobre variación terminológica. En el proyecto que se llevó a cabo en el centro de investigación CVC del Erasmus University College Brussels ${ }^{1}$, se elaboró un método con el fin de estudiar y comparar la variación terminológica en textos fuente y sus traducciones (Kerremans, 2010). En el proyecto que se realizó en el grupo de investigación IULATERM de la Universitat Pompeu Fabra², se adoptó un método para estudiar y describir la motivación cognitiva de la variación terminológica (Fernández-Silva, Freixa et al., 2009).

En la sección 2, resumiremos ambos proyectos. La metodología elaborada en el estudio piloto se describirá en la sección 3. En primer lugar, discutimos cómo se compiló el corpus (sección 3.1.) y cómo identificamos los términos fuente (sección 3.2.) y sus traducciones (sección 3.3.). A partir del análisis cognitivo de los términos fuente y sus traducciones, se introducen dos medidas en esta metodología: por un lado, la «distancia cognitiva» 
La variación terminológica en textos fuente y sus traducciones: un estudio piloto

entre los términos fuente y sus traducciones y, por otro lado, el «índice de variación interlingüística» de cada grupo de variantes terminológicas. Estas nociones se explicarán con más detalle en la sección 3.4. Después de discutir la metodología, presentaremos los resultados de nuestro estudio piloto en la sección 4. Estos resultados se discutirán en la sección 5 y, por último, en la sección 6 presentaremos nuestras conclusiones.

\section{Marco de la investigación}

En esta sección, resumiremos los proyectos de investigación del CVC (sección 2.1.) e IULATERM (sección 2.2.) y discutiremos algunas nociones esenciales derivadas en parte de estos dos proyectos para comparar cómo se refleja en las traducciones las elecciones de términos motivados cognitivamente en los textos fuente.

\subsection{Motivaciones cognitivas para la variación terminológica}

El proyecto que se llevó a cabo en el grupo de investigación IULATERM tiene como objetivo describir el comportamiento cognitivamente motivado de la variación terminológica. Partiendo de la premisa de que la formación de términos tiene una motivación (Guiraud, 1978; Kocourek, 1991; Sager, 1997 y Myking, 2009), se afirma que la variación terminológica es el resultado de múltiples motivaciones que tienen lugar en el proceso de denominación (Freixa, Fernández-Silva et al., 2008). Algunas de estas motivaciones se sitúan en el nivel del sistema de terminología, mientras que otras se sitúan en el nivel de uso. El tipo de UC que se nombra en un campo y la lengua que se emplea son factores que influyen en la elección de términos a nivel sistémico (Kageura, 2002); pero la denominación también se ve afectada por factores contextuales.

Los factores contextuales que se han estudiado en relación con la variación terminológica se organizan según diferentes niveles. El nivel cognitivo está relacionado con la perspectiva desde la cual un experto se acerca a la UC en una situación particular, lo que determinará qué características de la UC enfatiza el experto. Autores como Temmerman (2000) y Fernández-Silva, Freixa et al. (2009) han investigado este tema y sugieren que la elección de un término es influenciada por el campo de especialización de los autores. El nivel comunicativo implica las circunstancias de la producción y recepción del mensaje. Freixa (2002), por ejemplo, observó que el nivel de especialización de un texto determina el grado y los tipos de variación terminológica. Por último, la variación de términos también se ha estudiado a nivel discursivo: Collet (2004), por ejemplo, ha demostrado que los términos están sujetos a transformaciones formales y estructurales cuando se insertan en un entorno discursivo, dando lugar a diferentes tipos de variantes condicionadas por el contexto.

Con el fin de describir los patrones y las regularidades de la variación terminológica, se elaboró una metodología en la que se analizan los grupos de variantes terminológicas que se refieren a la misma UC con respecto a las características de la UC que estas enfatizan. Esta metodología es respaldada por importantes trabajos sobre formación de términos que sugieren que los términos a menudo reflejan las características más relevantes de la 
La variación terminológica en textos fuente y sus traducciones: un estudio piloto

UC (Kocourek, 1991; Sager, 1997). Hemos adoptado el análisis empleado por Kageura (2002) para describir los patrones de formación de términos motivados cognitivamente. En este análisis, la formación de términos se entiende como la especificación de conceptos dentro de una clase conceptual, representada por el núcleo, por medio de modificaciones representadas por los modificadores. Por lo tanto, el contenido del término se interpreta como una combinación de conceptos dentro del sistema conceptual general del campo. Estos conceptos se reflejan en los diferentes elementos constituyentes que forman un término.

La Tabla 2 muestra, a partir de un ejemplo de un texto fuente de nuestro estudio piloto, cómo cada elemento constituyente de un término se identifica primero como «núcleo» o como «modificador».

\begin{tabular}{|c|c|c|c|}
\hline Etiqueta UC & Lemas en gallego & Núcleo & Modificador \\
\hline \multirow{3}{*}{ AXENTE_ } & axente polucionante & axente & polucionante \\
\cline { 2 - 4 } POLUCIONANTE & axente contaminante & axente & contaminante \\
\cline { 2 - 4 } & componente tóxica & componente & tóxica \\
\hline
\end{tabular}

Tabla 2. Identificación del núcleo y del modificador

Después de identificar los elementos constituyentes, el siguiente paso es vincular cada elemento a su correspondiente concepto dentro del campo. Cada concepto se caracteriza según la categoría conceptual a la que pertenece y la característica distintiva que se refleja en su forma. En el ejemplo de la Tabla 2, todos los elementos de núcleo representan conceptos de sustancia, y se diferencian por la característica distintiva elegida para nombrarlo: la agentividad en axente (agent [agente]) o la estructura en componente (componente [componente]). Los modificadores muestran una cualidad o una actividad que caracteriza esta sustancia (Tabla 3).

\begin{tabular}{|c|c|c|c|}
\hline \multicolumn{2}{|c|}{ Información lingüística } & \multicolumn{2}{|c|}{ Información conceptual } \\
\hline Núcleo & Modificador & Núcleo & Modificador \\
\hline axente & polucionante & SUSTANCIA (POR ACCIÓN) & ACTIVIDAD \\
\hline axente & contaminante & SUSTANCIA (POR ACCIÓN) & ACTIVIDAD \\
\hline componente & tóxica & SUSTANCIA (POR ESTRUCTURA) & CALIDAD \\
\hline
\end{tabular}

Tabla 3. Identificación de la información conceptual del núcleo y del modificador

En nuestro estudio piloto, este método de análisis se adoptó para comparar las elecciones motivadas cognitivamente entre los términos fuente y sus traducciones; en particular, buscaremos qué aspectos de la UC tienen mayor preferencia en la elección de términos en los textos fuente y examinaremos si se respeta o no la 
La variación terminológica en textos fuente y sus traducciones: un estudio piloto misma motivación cognitiva en las traducciones. La forma en que se lleva a cabo este análisis se explica con más detalle en la sección 3.4.

\section{Metodología}

En nuestro estudio piloto, se examinó la variación terminológica en tres artículos científicos en gallego que abordan los efectos económicos de los desastres ambientales en la pesca y sus traducciones al inglés. En primer lugar, se realizó un estudio cuantitativo en el que se comparó el número de términos únicos en cada texto fuente con el número de traducciones únicas de estos términos. Luego, cada combinación única de un término fuente y su equivalente de traducción se sometieron a un análisis cualitativo con el fin de examinar posibles diferencias conceptuales entre el término fuente y su traducción.

Primero, discutiremos los criterios que se adoptaron para compilar el corpus y también proporcionaremos una breve descripción de los tres textos (sección 3.1.). Luego, explicaremos cómo se realizó el análisis de términos en los textos fuente (sección 3.2.). En la sección 3.3., nos centraremos en el análisis de los equivalentes de los términos fuente. También mostraremos cómo los términos fuente y sus correspondientes traducciones se anotaron de forma semiautomática en los textos paralelos. Por último, discutiremos los tipos de análisis que se llevaron a cabo en los datos anotados (sección 3.4.).

\subsection{Compilación del corpus}

Los textos de este estudio piloto se seleccionaron de un corpus más grande utilizado en el proyecto de investigación de IULATERM (Fernández-Silva, Freixa et al., 2009). Con el fin de garantizar que los textos seleccionados fueran comparables, estos se eligieron según los siguientes criterios:

a. Lengua: los textos fuente fueron escritos originalmente en gallego y luego traducidos al inglés.

b. Tema: todos los textos abordan las consecuencias económicas de los derrames de petróleo en el sector pesquero gallego.

c. Tipo de texto: todos los textos son artículos científicos, publicados en actas de congresos o revistas internacionales.

d. Área de estudio: todos los textos están relacionados con el área de estudio de la economía aplicada.

Las razones para elegir textos de un proyecto existente también eran de naturaleza práctica. En el momento de comenzar el estudio piloto, estábamos familiarizados con el campo y la terminología de la pesca costera y la acuicultura, lo cual es necesario para llevar a cabo el análisis cognitivo ( $c f$. sección 3.4.). Además, teníamos información sobre la génesis de los textos y sus traducciones, lo que resultó ser de primordial importancia para interpretar de forma correcta los resultados, como explicaremos en la sección 5. La información textual y 
La variación terminológica en textos fuente y sus traducciones: un estudio piloto extralingüística importante sobre los textos fuente se resume en la Tabla 4.

\begin{tabular}{|c|c|c|c|c|c|c|c|}
\hline Referencia & $\begin{array}{l}\text { Área de } \\
\text { estudio }\end{array}$ & $\begin{array}{l}\text { Tipo de } \\
\text { texto }\end{array}$ & Año & $\begin{array}{l}\text { Formato de } \\
\text { publicación }\end{array}$ & Audiencia & $\begin{array}{c}\text { Nivel de } \\
\text { especialización }\end{array}$ & $\begin{array}{c}\text { Recuento } \\
\text { de } \\
\text { palabras }\end{array}$ \\
\hline SG & $\begin{array}{c}\text { economía } \\
\text { aplicada }\end{array}$ & $\begin{array}{l}\text { artículo } \\
\text { científico }\end{array}$ & 2004 & $\begin{array}{l}\text { actas de } \\
\text { congreso }\end{array}$ & $\begin{array}{c}\text { expertos en } \\
\text { economía } \\
\text { marina y } \\
\text { ciencias } \\
\text { marinas }\end{array}$ & $\begin{array}{c}\text { altamente } \\
\text { especializado }\end{array}$ & 4166 \\
\hline GN & $\begin{array}{c}\text { economía } \\
\text { aplicada }\end{array}$ & $\begin{array}{l}\text { artículo } \\
\text { científico }\end{array}$ & 2004 & $\begin{array}{l}\text { actas de } \\
\text { congreso }\end{array}$ & $\begin{array}{c}\text { expertos en } \\
\text { economía } \\
\text { marina y } \\
\text { ciencias } \\
\text { marinas }\end{array}$ & $\begin{array}{c}\text { altamente } \\
\text { especializado }\end{array}$ & 8546 \\
\hline $\mathrm{DC}$ & $\begin{array}{c}\text { economía } \\
\text { aplicada }\end{array}$ & $\begin{array}{l}\text { artículo } \\
\text { científico }\end{array}$ & 2007 & $\begin{array}{l}\text { actas de } \\
\text { congreso }\end{array}$ & $\begin{array}{c}\text { expertos } \\
\text { del área, } \\
\text { pescadores, } \\
\text { empresarios }\end{array}$ & $\begin{array}{c}\text { altamente } \\
\text { especializado }\end{array}$ & 8960 \\
\hline
\end{tabular}

Tabla 4. Información sobre los textos fuente

Estos tres textos fuente se alinearon con sus traducciones a nivel de oración (siempre que esto fuera posible). Los textos paralelos resultantes se utilizaron para identificar y anotar los términos fuente (sección 3.2.) y sus traducciones (sección 3.3.).

\subsection{Identificación de los términos de la lengua fuente}

El análisis del texto fuente y la extracción de términos se llevaron a cabo con la ayuda de la herramienta de análisis textual TextStat. ${ }^{3}$ Luego, la lista de términos resultante se complementó con variantes de términos que se extrajeron de forma manual de los textos. A los términos que se consideraron variantes de la misma UC se les asignó la misma etiqueta UC (sección 2.1.). La lista de términos resultante, así como las relaciones de equivalencia entre variantes terminológicas, fueron validadas por expertos del área (Fernández-Silva, Freixa et al., 2009).

La lista de términos se utilizó para buscar y resaltar automáticamente términos en los contextos de la lengua fuente de los textos paralelos. Cada término correspondiente se colocó entre etiquetas de identificación. La etiqueta de identificación proporciona dos tipos de información: por un lado, vincula el término a una UC al mostrar la etiqueta única de UC; por otro lado, asigna un número único para cada término por oración. Este número se utiliza 
La variación terminológica en textos fuente y sus traducciones: un estudio piloto para ubicar la traducción del término fuente en la oración meta (sección 3.3.). Esto es crucial si una UC se expresa más de una vez en una oración fuente.

El siguiente ejemplo ilustra cómo los términos fuente en los textos del corpus se anotaron de forma semiautomática por medio de un programa escrito en Perl que se desarrolló en el marco del proyecto de investigación CVC (sección 2.1.). Esta muestra se tomó del texto fuente «GN» (Tabla 3).

[...] pode ver-se retardada a velocidade de migración, con todo o que iso implica na $\| 2$-PESCA $\|$ pesca $\|2-P E S C A\|$ e supervivéncia da espécie, xa que se o migrante se desvia do seu lugar habitual de freza, por mor dun $\| 1$-AXENTE

POLUCIONANTE||axente polucionante||1-AXENTE_POLUCIONANTE||, a povoación pode verse afectada dun xeito esaxerado.

\subsection{Identificación de equivalentes de traducción}

Después de la anotación de los términos fuente, se buscaron las traducciones de estos términos en las oraciones meta en cada texto paralelo. Una traducción recibía la misma etiqueta de identificación que su correspondiente término en el texto fuente, como se observa en la siguiente muestra tomada del texto «GN» (Tabla 5).

\begin{tabular}{|c|c|}
\hline Gallego & $\begin{array}{l}{[\ldots] \text { pode ver-se retardada a velocidade de migración, con }} \\
\text { todo o que iso implica na } \| 2 \text {-PESCA } \| \text { pesca\||2-PESCA } \| \text { e } \\
\text { supervivéncia da espécie, xa que se o migrante se desvia } \\
\text { do seu lugar habitual de freza, por mor dun } \| 1 \text {-AXENTE } \\
\text { POLUCIONANTE\|axente polucionante } \| 1-A X E N T E \\
\text { POLUCIONANTE\|, a povoación pode verse afectada } \\
\text { dun xeito esaxerado. }\end{array}$ \\
\hline Inglés & 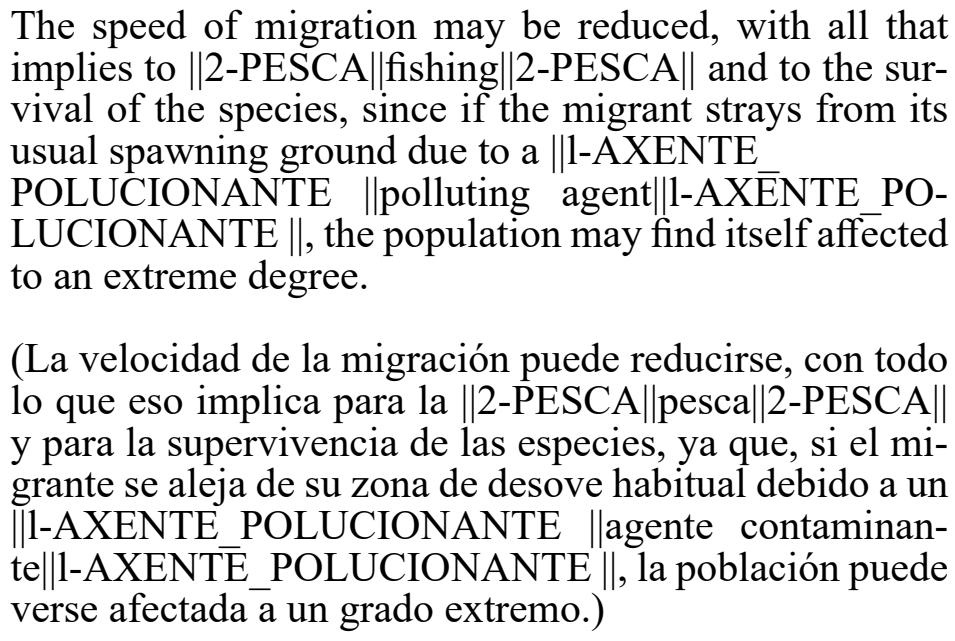 \\
\hline
\end{tabular}

Tabla 5. Fragmento anotado de los textos fuente y meta alineados

En caso de que el término fuente no estuviera traducido en el texto meta, la etiqueta de identificación única del término fuente se agregaba al final de la oración meta. De esa manera, la unidad de traducción se resaltaba como 
La variación terminológica en textos fuente y sus traducciones: un estudio piloto una «traducción cero», lo que resultaba del proceso de omisión.

La combinación de una etiqueta UC, el término fuente y su equivalente de traducción se denomina «unidad de traducción». En la Tabla 6, se muestran ejemplos de unidades de traducción derivadas de uno de los textos paralelos en nuestro corpus.

\begin{tabular}{|c|c|c|c|c|}
\hline $\begin{array}{c}\text { Unidad de } \\
\text { comprensión }\end{array}$ & $\begin{array}{c}\text { Lema en } \\
\text { gallego }\end{array}$ & $\begin{array}{c}\text { Ocurrencia en } \\
\text { gallego } \\
\end{array}$ & Lema en inglés & $\begin{array}{c}\text { Ocurrencia en } \\
\text { inglés }\end{array}$ \\
\hline AXENTE_POLUCIONANTE & $\begin{array}{c}\text { axente } \\
\text { polucionante }\end{array}$ & $\begin{array}{c}\text { axente } \\
\text { polucionante }\end{array}$ & $\begin{array}{c}\text { polluting } \\
\text { agent (agente } \\
\text { contaminante) }\end{array}$ & $\begin{array}{c}\text { polluting } \\
\text { agents (agentes } \\
\text { contaminantes) }\end{array}$ \\
\hline AXENTE_POLUCIONANTE & $\begin{array}{c}\text { axente } \\
\text { contaminante }\end{array}$ & $\begin{array}{c}\text { axentes } \\
\text { contaminantes }\end{array}$ & $\begin{array}{c}\text { pollutant } \\
\text { (contaminante) }\end{array}$ & $\begin{array}{c}\text { pollutants } \\
\text { (contaminantes) }\end{array}$ \\
\hline AXENTE_POLUCIONANTE & $\begin{array}{l}\text { componente } \\
\text { tóxica }\end{array}$ & $\begin{array}{l}\text { componentes } \\
\text { tóxicas }\end{array}$ & $\begin{array}{l}\text { toxic component } \\
\text { (componente } \\
\text { tóxico) }\end{array}$ & $\begin{array}{c}\text { toxic components } \\
\text { (componentes } \\
\text { tóxicos) }\end{array}$ \\
\hline
\end{tabular}

Tabla 6. Ejemplos de unidades de traducción

\subsection{Análisis de la variación terminológica}

De una lista de 629 términos que se refieren a 484 UC, solo se conservaron aquellas que aparecían más de una vez en cualquiera de los textos, ya que nuestro análisis solo se centraba en las UC que se caracterizaban por tener variación terminológica en los textos fuente. Las unidades de traducción se extrajeron automáticamente de los corpus anotados y se copiaron a un formato de tabla. El formato se ilustra mediante la Tabla 7 en la que los ejemplos se tomaron del texto «DC». Cada unidad de traducción en la tabla se complementó con una especificación de los lemas en las lenguas fuente y meta. Además de eso, también se calculó con qué frecuencia aparecía una unidad de traducción particular en cada texto. 
La variación terminológica en textos fuente y sus traducciones: un estudio piloto

\begin{tabular}{|c|c|c|c|c|c|}
\hline Etiqueta UC & $\begin{array}{c}\text { Lema en } \\
\text { gallego }\end{array}$ & $\begin{array}{c}\text { Ocurrencia } \\
\text { en gallego }\end{array}$ & Lema en inglés & $\begin{array}{c}\text { Ocurrencia } \\
\text { en inglés }\end{array}$ & Frecuencia \\
\hline ESPECIES_COMERCIAIS & $\begin{array}{c}\text { especies } \\
\text { comerciais }\end{array}$ & $\begin{array}{c}\text { especies } \\
\text { comerciais }\end{array}$ & $\begin{array}{c}\text { affected species } \\
\text { (especies } \\
\text { afectadas) }\end{array}$ & $\begin{array}{c}\text { affected } \\
\text { species } \\
\text { (especies } \\
\text { afectadas) }\end{array}$ & 1 \\
\hline ESPECIES_COMERCIAIS & especies & especies & $\begin{array}{c}\text { market resource } \\
\text { comerciais }\end{array}$ & $\begin{array}{c}\text { market } \\
\text { comerciais de } \\
\text { resources } \\
\text { (recursos de } \\
\text { mercado) }\end{array}$ & 1 \\
\hline
\end{tabular}

Tabla 7. Ejemplo de datos finales

Luego, los resultados en la tabla se utilizaron para llevar a cabo tres tipos de análisis. En un primer análisis cuantitativo, examinamos la variación terminológica que se presentaba en los textos fuente. El objetivo de este análisis era verificar cuántas UC en los textos fuente se caracterizaron por la variación terminológica. Con el fin de examinar si esta variación también se reflejaba en las traducciones especializadas y de qué manera esto ocurría, se llevaron a cabo dos análisis adicionales: por un lado, un análisis cuantitativo y una comparación del número de expresiones (para cada UC) encontradas tanto en el texto fuente como en el meta y, por otro lado, un análisis cognitivo cualitativo de las unidades de traducción.

El análisis cognitivo presentado en la sección 2.2. nos permitió comparar la información conceptual entre los términos fuente y sus traducciones. Un ejemplo de esta comparación se muestra en la Tabla 8 . Se debe tener en cuenta que las columnas se organizan según la posición de los núcleos y los modificadores en gallego, lo que resulta en una inversión de elementos en los compuestos adjetivo + sustantivo en inglés. El ejemplo muestra que, en dos de tres traducciones, el patrón conceptual se reflejó literalmente, lo que significa que el traductor respetó la motivación cognitiva del autor del texto fuente. El término fuente axente contaminante y su traducción pollutant (contaminante) difieren en términos de información conceptual: mientras que en el término fuente la actividad se expresa en el modificador y el núcleo enfatiza el papel activo de la sustancia, la traducción nombra la sustancia a partir de la actividad, dejando de lado su papel de agente. 
La variación terminológica en textos fuente y sus traducciones: un estudio piloto

\begin{tabular}{|c|c|c|c|}
\hline Términos (LF) & $\begin{array}{c}\text { patrón cognitivo } \\
\text { [NÚCLEO + } \\
\text { MODIFICADOR] }\end{array}$ & Términos (LM) & $\begin{array}{c}\text { Patrón cognitivo } \\
\text { [NÚCLEO + MODIFICADOR] }\end{array}$ \\
\hline $\begin{array}{c}\text { axente } \\
\text { polucionante }\end{array}$ & $\begin{array}{c}\text { [SUSTANCIA (POR } \\
\text { ACCIÓN) + } \\
\text { ACTIVIDAD] }\end{array}$ & $\begin{array}{l}\text { polluting agent (agente } \\
\text { contaminante) }\end{array}$ & $\begin{array}{c}\text { [SUSTANCIA (POR ACCIÓN) }+ \\
\text { ACTIVIDAD }]\end{array}$ \\
\hline $\begin{array}{c}\text { axente } \\
\text { contaminante }\end{array}$ & $\begin{array}{c}\text { [SUSTANCIA (POR } \\
\text { ACCIÓN) }+ \\
\text { ACTIVIDAD] }\end{array}$ & $\begin{array}{c}\text { pollutant } \\
\text { (contaminante) }\end{array}$ & SUSTANCIA (POR ACTIVIDAD) \\
\hline $\begin{array}{l}\text { componente } \\
\text { tóxica }\end{array}$ & $\begin{array}{c}\text { [SUSTANCIA (POR } \\
\text { ESTRUCTURA) }+ \\
\text { CALIDAD] }\end{array}$ & $\begin{array}{l}\text { toxic component } \\
\text { (componente tóxico) }\end{array}$ & $\begin{array}{c}\text { [SUSTANCIA (POR ESTRUCTURA) }+ \\
\text { CALIDAD }]\end{array}$ \\
\hline
\end{tabular}

Tabla 8. Comparación de la información cognitiva

Con base en este tipo de comparación, pudimos asignar de forma manual un valor a cada unidad de traducción con el fin de calificar la distancia cognitiva (es decir, las diferencias en la información conceptual) entre el término fuente y su traducción. Un valor de 0 indica «sin distancia cognitiva» en el sentido de que no hay diferencia en la información conceptual entre el término fuente (p. ej., axente polucionante) y su equivalente de traducción (p. ej., polluting agent [agente contaminante]). Un valor de 0,5 significa una «distancia cognitiva parcial»; este valor se asigna a una unidad de traducción si hay una coincidencia parcial en la información conceptual entre el término fuente y su traducción. Por ejemplo, el caso de axente contaminante y su traducción pollutant (contaminante) se clasificarían de esa manera. Cuando una unidad de traducción se califica como 1 significa que, respecto a la información conceptual, existe un grado mínimo de correspondencia entre el término fuente y su traducción. Un ejemplo sería axente económico (economic agent [agente económico]) que se tradujo en el texto en inglés «DC» como fishermen (pescador). En este caso, la única similitud es que los conceptos en la posición de núcleos pertenecen a la misma categoría amplia de humanos (entidades animadas), pero la característica distintiva, así como la actividad relacionada con el profesional, no coinciden.

Con base en las distancias cognitivas de las unidades de traducción y la frecuencia de la unidad de traducción en cada texto paralelo, calculamos el índice de variación interlingüística (IVI). El IVI es una medida entre 0 y 1 que indica cómo se transfiere una UC del texto fuente al texto meta. Si esta medida está cerca de 0 , significa que, en general, se realizó una traducción más directa o literal con respecto a la traducción de una UC en particular. Una medida del IVI que esté más cerca de 1 indica una traducción más libre. La medida del IVI es un promedio. Es el resultado de la suma de las distancias cognitivas ponderadas (es decir, la distancia cognitiva 
La variación terminológica en textos fuente y sus traducciones: un estudio piloto de una unidad de traducción multiplicada por la frecuencia de la unidad de traducción) que luego se divide por el número total de unidades de traducción para cada UC. Esto se ilustra mediante el ejemplo de la Tabla 9.

\begin{tabular}{|c|c|c|c|c|c|}
\hline UC & Lema (TF) & Lema (TM) & Frecuencia & $\begin{array}{c}\text { Distancia } \\
\text { cognitiva }\end{array}$ & $\begin{array}{c}\text { Distancia } \\
\text { cognitiva } \\
\text { ponderada }\end{array}$ \\
\hline $\begin{array}{c}\text { AXENTE_- } \\
\text { POLUCIONANTE }\end{array}$ & \begin{tabular}{c} 
polluting \\
polucionante \\
\cline { 2 - 6 }
\end{tabular} & $\begin{array}{c}\text { agent (agente } \\
\text { contaminante) }\end{array}$ & 1 & 0 & 0 \\
& $\begin{array}{c}\text { axente } \\
\text { contaminante }\end{array}$ & $\begin{array}{c}\text { pollutant } \\
\text { (contaminante) }\end{array}$ & 1 & 0,5 & 0,5 \\
\cline { 2 - 6 } & $\begin{array}{c}\text { componente } \\
\text { tóxica } \\
\text { (componente } \\
\text { tóxico) }\end{array}$ & 1 & 0 & 0 \\
\hline
\end{tabular}

Tabla 9. Cálculo del Índice de Variación Interlingüistica (IVI)

El IVI nos permite evaluar las consecuencias cognitivas de las elecciones de equivalentes y describir mejor las diferencias entre los textos fuente y sus traducciones. Esta medida no solo nos permite ver si el patrón de variación para cada UC en el texto fuente se refleja o no en la traducción, sino que también tiene en cuenta hasta qué punto el traductor respetó el punto de vista cognitivo reflejado en la elección de terminología por parte del autor en el texto fuente.

\section{Resultados}

Los resultados presentados en esta sección solo se basan en las UC que aparecen más de una vez en cada texto (sección 3.4.). A algunas de estas UC se hizo referencia solo con un término, mientras que otras se caracterizaban por tener múltiples denominaciones. Dado que las UC que aparecían solo una vez se excluyeron de nuestro análisis, solo se mantuvieron 41 de las 59 UC que encontramos en el texto «DC» (es decir, 69,5\%); en el texto «GN», 48 de las $74 \mathrm{UC}$ se consideraron relevantes (es decir, 64,9\%) y, en el texto «SG», se retuvieron 40 de las $53 \mathrm{UC}$ (es decir, 75,5\%). 
La variación terminológica en textos fuente y sus traducciones: un estudio piloto

\subsection{Variación terminológica en los textos fuente}

En esta sección, examinamos la variación terminológica que se encontró en los textos fuente. Los resultados del análisis cuantitativo se muestran en la Tabla 10. La categoría «Total» indica el número de UC que aparecen al menos dos veces en el mismo texto fuente. La categoría «=1» es un subconjunto de la categoría «Total» y representa el número de UC que se caracterizan por tener un solo término. La categoría «>1» muestra el subconjunto de UC que se caracterizan por tener más de un término en el texto fuente.

\begin{tabular}{|c|c|c|c|c|c|c|}
\cline { 2 - 7 } \multicolumn{1}{c|}{} & \multicolumn{3}{c|}{$\begin{array}{c}\text { Ocurrencias en los } \\
\text { textos }\end{array}$} & \multicolumn{3}{c|}{ Lemas } \\
\cline { 2 - 7 } \multicolumn{1}{c|}{} & Total & $=\mathbf{1}$ & $>\mathbf{1}$ & Total & $=\mathbf{1}$ & $>\mathbf{1}$ \\
\hline DC & 41 & 19 & 22 & 41 & 20 & 21 \\
\hline GN & 48 & 14 & 34 & 48 & 15 & 33 \\
\hline SG & 40 & 9 & 31 & 40 & 10 & 30 \\
\hline
\end{tabular}

Tabla 10. UC que aparecen más de una vez en los textos

De los resultados de la Tabla 10, podemos deducir que 22 UC de las 41 que aparecen más de una vez en el texto «DC» se caracterizan por tener más de un término ( $c f . «>1 »)$.

Esto corresponde aproximadamente al 53,7 \% de ocurrencias, o al 51,2 \% de lemas. En el caso del texto «GN», encontramos que el 70,8 \% de las UC se caracterizan por tener más de un término $(68,8 \%$ después de la lematización). Por último, en el texto «SG», esta cantidad corresponde al 80 \% (75\% después de la lematización). Estos resultados muestran que el texto fuente «DC» se caracteriza por presentar una menor variación terminológica que los otros dos textos.

Las pequeñas diferencias en los números entre la variación terminológica basada en ocurrencias en los textos y lemas muestran que la variación morfosintáctica (es decir, formas de palabras singulares vs. plurales) no parece ser la causa principal de variación, ni en los textos fuente en gallego ni en sus traducciones al inglés. La mayoría de las UC que se caracterizaban por tener múltiples denominaciones consisten en términos que diferían parcial o completamente entre sí en términos de realización superficial. Encontramos UC a las cuales se hizo referencia por medio de un término más general y uno de sus hipónimos. La UC //ECOSISTEMA//, por ejemplo, se caracteriza por ecosistema (ecosystem [ecosistema]) y el término más específico ecosistema mariño (marine ecosystem [ecosistema marino]) en el texto «DC». En otros casos, encontramos UC en las que un elemento constituyente de un término se mantiene mientras que el otro varía. Ejemplos de esto son: efecto da catástrofe (effect of the catastrophe [efecto de la catástrofe]) y efecto do derramo (effect of the spill [efecto del derrame]) en el texto «SG» o zona afectada (affected zone [zona afectada]) y zona polucionada (polluted zone [zona contaminada]) en el texto «GN». En todos los demás casos, el término completo se reemplaza por otro, ya sea por un sinónimo lingüístico o por un término que se utiliza como tal en el contexto específico. Este es, por ejemplo, el caso de la UC // 
La variación terminológica en textos fuente y sus traducciones: un estudio piloto

ACTIVIDADE_PESQUEIRA// que se caracteriza por los siguientes lemas en el texto «GN»: actividade pesqueira (fishing activity [actividad pesquera]), captura (catch [captura]), explotación (exploitation [explotación]) y pesca (fishing [pesca]).

\subsection{Variación terminológica en los textos fuente y sus traducciones}

Una comparación de la variación interlingüística en los textos fuente y meta se presenta en los resultados de la Tabla 11. Se debe tener en cuenta que los resultados de la categoría «> 1» (Tabla 10) se toman como punto de partida para la comparación porque decidimos centrarnos solo en UC que se caracterizaran por tener múltiples denominaciones en el texto fuente.

\begin{tabular}{|c|c|c|c|c|}
\cline { 2 - 5 } \multicolumn{1}{c|}{} & \multicolumn{4}{c|}{ Lemas ('> 1') } \\
\cline { 2 - 5 } \multicolumn{1}{c|}{} & $\mathbf{U C}$ & $\mathbf{G a}<\mathbf{E n}$ & $\mathbf{G a}=\mathbf{E n}$ & $\mathbf{G a}>\mathbf{E n}$ \\
\hline $\mathbf{D C}$ & 21 & 6 & 12 & 3 \\
\hline $\mathbf{G N}$ & 33 & 6 & 18 & 9 \\
\hline SG & 30 & 8 & 15 & 7 \\
\hline
\end{tabular}

Tabla 11. Comparación de la variación en los textos fuente y meta

En esta tabla, las UC que tienen más términos fuente únicos en comparación con las traducciones únicas se clasificaron como «Ga $>$ En». Si se encontraban más traducciones en el texto meta, la UC se clasificaba como «Ga $<$ En». Por último, una UC se clasificaba como «Ga $=$ En», si se encontraba el mismo número de expresiones únicas en los textos fuente y meta.

De los resultados de la Tabla 11, deducimos que la mayoría de las UC tienden a tener el mismo número de lexicalizaciones en los textos fuente y meta: 57,1\% (texto «DC»), 54,5\% (texto «GN») y $50 \%$ (texto «SG»). Esto muestra que, en general, la variación que ocurre en los textos fuente también está presente en los textos meta. El bajo número de UC que entra en la categoría «Ga $>$ En» en el texto «DC» $(14,3 \%)$ en comparación con el texto «GN» $(27,3 \%)$ y el texto «SG» $(23,3 \%)$ puede implicar que la traducción de términos en este texto parece desviarse más de su original en comparación con las otras dos traducciones, lo cual se explorará más a fondo en el análisis cualitativo (sección 5.3.).

Para algunas UC que tienen más variantes en gallego que en inglés («Ga $>$ En»), se identificaron situaciones de variación gráfica. Por ejemplo, en el texto «DC» encontramos los términos producción mexilloeira y produzón mexilloeira, que se tradujeron como mussel production (producción de mejillones). La existencia de una mayor cantidad de variantes ortográficas en los textos en gallego en comparación con los textos en inglés puede deberse 
La variación terminológica en textos fuente y sus traducciones: un estudio piloto al hecho de que el uso del gallego como una lengua para la comunicación especializada no es muy amplio y, por lo tanto, los expertos que escriben en gallego pueden no estar familiarizados con la correcta ortografía de algunos términos de su área. Además, la variación ortográfica en el texto «DC» se debe a la presencia de dos normas ortográficas para la lengua gallega: la Norma Reintegracionista y la Norma Oficial. ${ }^{4}$

Algunas UC que tienen más variantes en inglés que los textos fuente en gallego («En > Ga») hacen referencia a realidades específicas del sector pesquero gallego y podrían no tener un equivalente directo y completo en otras lenguas (Tabla 12). Por ejemplo, la noción de //MARISQUEO//, que se refiere a la actividad de recolección de mariscos, es una actividad muy importante en Galicia y se considera legalmente como una modalidad de pesca. Sin embargo, en otros países, esta actividad ha sido abandonada y los mariscos solo se producen por cultivo, por lo tanto, se considera como una modalidad de acuicultura. Esto explica la variedad de expresiones encontradas en los textos meta para traducir la actividad //ACTIVIDADE_MARISQUEIRA//, el sector // SECTOR_MARISQUEIRO// o el profesional//MARISCADOR//. En la UC //ACTIVIDADE_PESQUEIRA// [//actividad pesquera//], también observamos la estrategia utilizada por el traductor para incluir la recolección de mariscos en el texto meta. Otras nociones arraigadas culturalmente de equivalencia inexacta son el sector de cultivo de mejillones //SECTOR_MEXILLOEIRO// o los mercados específicos donde se vende pescado en una subasta //LONXA//.

\begin{tabular}{|c|c|c|c|}
\hline $\mathbf{U C}$ & $\begin{array}{c}\text { Ocurrencias } \\
\text { en textos en } \\
\text { gallego }\end{array}$ & Ocurrencias en textos en inglés & Texto \\
\hline $\begin{array}{l}\text { ACTIVIDADE_ } \\
\text { MARISQUEIRA }\end{array}$ & marisqueo & $\begin{array}{l}\text { shellfish collecting, shellfish-collecting, } \\
\text { shellfish-collecting activity (colecta } \\
\text { de mariscos, recolección de mariscos, } \\
\text { actividad de recolección de mariscos) }\end{array}$ & $\mathrm{DC}$ \\
\hline $\begin{array}{l}\text { ACTIVIDADE_ } \\
\text { MARISQUEIRA }\end{array}$ & $\begin{array}{l}\text { actividade } \\
\text { marisqueira, } \\
\text { marisqueo }\end{array}$ & $\begin{array}{l}\text { shellfish harvesting activity, harvesting, } \\
\text { shellfish harvesting (actividad de } \\
\text { cosecha de mariscos, cosecha, cosecha } \\
\text { de mariscos) }\end{array}$ & GN \\
\hline $\begin{array}{c}\text { SECTOR_- } \\
\text { MARISQUEIRO }\end{array}$ & marisqueo & $\begin{array}{c}\text { shellfish collecting, shellfish-collecting } \\
\text { (recolección de mariscos, colecta de } \\
\text { mariscos) }\end{array}$ & $\mathrm{DC}$ \\
\hline
\end{tabular}


La variación terminológica en textos fuente y sus traducciones: un estudio piloto

\begin{tabular}{|c|c|c|c|}
\hline $\begin{array}{c}\text { SECTOR }_{-} \\
\text {MARISQUEIRO }\end{array}$ & $\begin{array}{l}\text { marisqueo, } \\
\text { subsector de } \\
\text { marisqueo }\end{array}$ & $\begin{array}{c}\text { harvesting, shellfish harvesting, } \\
\text { subsector of shellfish harvesting } \\
\text { (cosecha, cosecha de mariscos, subsector } \\
\text { de la cosecha de mariscos) }\end{array}$ & GN \\
\hline MARISCADOR & $\begin{array}{c}\text { mariscadores, } \\
\text { productores }\end{array}$ & $\begin{array}{c}\text { persons engaged in mariculture, } \\
\text { shellfish harvesters, producers (personas } \\
\text { dedicadas a la maricultura, cosechadores } \\
\text { de mariscos, productores) }\end{array}$ & SG \\
\hline $\begin{array}{c}\text { ACTIVIDADE } \\
\text { PESQUEIRA }\end{array}$ & $\begin{array}{l}\text { actividade } \\
\text { pesqueira, } \\
\text { captura, } \\
\text { explotación, } \\
\text { pesca }\end{array}$ & $\begin{array}{l}\text { fishing and shellfish harvesting activity, } \\
\text { capture, catch, fishing or harvesting, } \\
\text { harvesting, fishing (actividad de } \\
\text { pesca y cosecha de mariscos, captura, } \\
\text { apresamiento, pesca o cosecha, cosecha, } \\
\text { pesca) }\end{array}$ & GN \\
\hline $\begin{array}{c}\text { SECTOR } \\
\text { MEXILLOEIRO }\end{array}$ & $\begin{array}{l}\text { sector } \\
\text { mexilloeiro, } \\
\text { subsector de } \\
\text { miticultura }\end{array}$ & $\begin{array}{l}\text { mussel culture sector, the mussel } \\
\text { harvesting sector, subsectors of } \\
\text { mussel culture (sector de cultivo de } \\
\text { mejillón, sector de cosecha de mejillón, } \\
\text { subsectores de cultivo de mejillón) }\end{array}$ & GN \\
\hline LONXA & lonxa & $\begin{array}{c}\text { market, auction market (mercado, } \\
\text { mercado de subastas) }\end{array}$ & $\mathrm{DC}$ \\
\hline
\end{tabular}

Tabla 12. Traducción de UC culturales específicas en la categoría «Ga $>$ En» 
La variación terminológica en textos fuente y sus traducciones: un estudio piloto

Algunos casos de disimetría entre el número de términos fuente y los equivalentes de traducción se explican por medio de procesos regulares de variación morfosintáctica existentes en cada lengua. Estos se emplean principalmente por razones estilísticas o preferencias de escritura; por lo tanto, el traductor puede sentirse libre de reducir o provocar este tipo de variación. La siguiente tabla muestra algunas de estas situaciones:

\begin{tabular}{|c|c|c|c|}
\hline UC & $\begin{array}{c}\text { Ocurrencias en textos } \\
\text { en gallego }\end{array}$ & Ocurrencias en textos en inglés & Texto \\
\hline CRIA & cria & seeding, seeds (siembra, cría) & $\mathrm{GN}$ \\
\hline MADURACIÓN & maduración & maturation, maturing (maduración, & $\mathrm{GN}$ \\
\hline $\begin{array}{c}\text { SECTOR_DA_- } \\
\text { PESCA }\end{array}$ & pesca & fisheries, fishing (pesca, pesquería) & $\mathrm{GN}$ \\
\hline $\begin{array}{c}\text { ZONA_DE_- } \\
\text { PROHIBICIÓN }\end{array}$ & $\begin{array}{c}\text { zonas de prohibición, } \\
\text { zonas prohibidas }\end{array}$ & prohibited zones (zonas prohibidas) & $\mathrm{SG}$ \\
\hline $\begin{array}{c}\text { PRODUCCIÓN_ } \\
\text { DE_MEXILLÓN }\end{array}$ & $\begin{array}{c}\text { producción de mexillón, } \\
\text { producción do mexillón }\end{array}$ & $\begin{array}{c}\text { mussel production (producción de } \\
\text { mejillones) }\end{array}$ & $\mathrm{SG}$ \\
\hline
\end{tabular}

Tabla 13. Variación morfosintáctica en los textos fuente y meta

Otros usos asimétricos se deben al uso de sinónimos completos en la lengua fuente o meta (Tabla 14). Al comparar estos términos en cada lengua no se ve ningún cambio con respecto a la información conceptual (sección 4.4.).

\begin{tabular}{|c|c|c|c|}
\hline UC & $\begin{array}{c}\text { Ocurrencias en } \\
\text { textos en gallego }\end{array}$ & $\begin{array}{c}\text { Ocurrencias en textos en } \\
\text { inglés }\end{array}$ & Texto \\
\hline ACÍDENTE & catástrofe & $\begin{array}{c}\text { catastrophe, disaster (catástrofe, } \\
\text { desastre) }\end{array}$ & DC \\
\hline CRU & fuel & fuel, oil (combustible, gasolina) & DC \\
\hline SOBRECAPTURA & sobrecaptura & $\begin{array}{c}\text { excess catch, over-catch } \\
\text { (captura en exceso, }\end{array}$ & GN \\
& & \begin{tabular}{c} 
sobrecaptura) \\
\hline
\end{tabular}
\end{tabular}


La variación terminológica en textos fuente y sus traducciones: un estudio piloto

\begin{tabular}{|c|c|c|c|}
\hline ZONA_AFECTADA & $\begin{array}{c}\text { zona afectada, zona } \\
\text { polucionada }\end{array}$ & $\begin{array}{c}\text { affected area, affected zone, } \\
\text { zone affected, area polluted } \\
\text { (área afectada, zona afectada, } \\
\text { zona perjudicada, área } \\
\text { contaminada) }\end{array}$ & GN \\
\hline EMPREGO & emprego & $\begin{array}{c}\text { employment, jobs (empleo, } \\
\text { trabajos) }\end{array}$ & SG \\
\hline FoRTE_ & forte contaminación & $\begin{array}{c}\text { heavy pollution, massive } \\
\text { pollution } \\
\text { (fuerte contaminación, } \\
\text { contaminación masiva) }\end{array}$ & SG \\
\hline ZoNA_AFECTADA & zonas afectadas & $\begin{array}{c}\text { affected zones, areas affected } \\
\text { (zonas afectadas, áreas } \\
\text { afectadas) }\end{array}$ & SG \\
\hline
\end{tabular}

Tabla 14. Sinónimos totales en los textos fuente y meta

\subsection{Análisis de la distancia cognitiva y del índice de variación interlingüistica}

En la Tabla 15, solo mostramos el promedio del IVI para cada texto.

\begin{tabular}{|c|c|c|c|c|c|c|c|c|c|}
\hline & \multirow[b]{2}{*}{ UC } & \multicolumn{2}{|c|}{$\begin{array}{c}\text { Distancia } \\
\text { cognitiva ' } 0 \text { ' }\end{array}$} & \multicolumn{2}{|c|}{$\begin{array}{c}\text { Distancia } \\
\text { cognitiva }(0,5,\end{array}$} & \multicolumn{2}{|c|}{$\begin{array}{c}\text { Distancia } \\
\text { cognitiva ' } 1 \text { ' }\end{array}$} & \multirow{2}{*}{$\begin{array}{l}\text { N. }{ }^{\circ} \text { total de } \\
\text { unidades de } \\
\text { traducción }\end{array}$} & \multirow[b]{2}{*}{ IVI } \\
\hline & & $\mathrm{N}^{\mathrm{o}}$ & $\%$ & N. ${ }^{\circ}$ & $\%$ & $\mathrm{~N}^{\circ}$ & $\%$ & & \\
\hline $\mathrm{DC}$ & 21 & 92 & 62,1 & 22 & 14,9 & 34 & 22,97 & 148 & 0,304 \\
\hline GN & 33 & 303 & 83,24 & 46 & 12,64 & 15 & 4,12 & 364 & 0,104 \\
\hline SG & 30 & 187 & 85,78 & 22 & 10,09 & 9 & 4,13 & 218 & 0,092 \\
\hline
\end{tabular}

Tabla 15. Resultados del Índice de Variación Interlingüística (IVI)

Como era de esperarse, la proporción de equivalentes con puntaje 0 es mayor en los tres textos, lo que confirma nuestra hipótesis de que las traducciones tienden a ser consistentes y reflejan lo más cerca posible las elecciones de términos de los textos fuente. De manera similar, el número de equivalentes con una distancia cognitiva de 0,5 , que corresponde a traducciones en las que la información cognitiva se superpone en parte con la del término fuente, también es bastante homogéneo en los tres textos. Sin embargo, la proporción de variantes que difiere del 
La variación terminológica en textos fuente y sus traducciones: un estudio piloto

texto fuente es notablemente alta en el texto «DC»; de hecho, su IVI es tres veces mayor en comparación con los otros dos textos. Esto muestra con claridad que la traducción del texto «DC» tiende a desviarse más del texto fuente; el traductor del texto «DC» adoptó un estilo de traducción más libre en comparación con los traductores de los otros dos textos. Esto se muestra, como ilustración, en el siguiente ejemplo: 
La variación terminológica en textos fuente y sus traducciones: un estudio piloto

\begin{tabular}{|c|c|c|c|c|}
\hline $\mathbf{U C}$ & $\begin{array}{c}\text { Ocurrencias en textos } \\
\text { en gallego }\end{array}$ & Ocurrencias en textos en inglés & Frec. & $\begin{array}{l}\text { Distancia } \\
\text { cognitiva }\end{array}$ \\
\hline \multirow{5}{*}{$\begin{array}{l}\text { EFECTO_DA_ } \\
\text { CATÁSTROFE }\end{array}$} & efectos da marea negra & $\begin{array}{l}\text { oil spill effects (efectos del derrame } \\
\text { de petróleo) }\end{array}$ & 1 & 0 \\
\hline & efectos do vertido & $\begin{array}{l}\text { effects of the disaster (efectos del } \\
\text { desastre) }\end{array}$ & 1 & 0,5 \\
\hline & efectos do vertido & spill (derrame) & 1 & 0,5 \\
\hline & $\begin{array}{c}\text { impacto da marea } \\
\text { negra }\end{array}$ & $\begin{array}{c}\text { ecological catastrophe (catástrofe } \\
\text { ecológica) }\end{array}$ & 1 & 1 \\
\hline & $\begin{array}{c}\text { impacto da marea } \\
\text { negra }\end{array}$ & oil spill (vertido de petróleo) & 1 & 0,5 \\
\hline
\end{tabular}

Tabla 16. Ejemplo de traducción libre en el texto «DC»

Observamos que tres términos fuente se tradujeron por cinco expresiones diferentes. Además, el patrón cognitivo recurrente en los términos fuente [RELACIÓN DE CONSECUENCIA + CAMBIO DE ESTADO] solo se respeta en la primera ocurrencia. La elección terminológica en la traducción muestra un claro cambio de perspectiva, el traductor enfatiza las dramáticas consecuencias ecológicas del incidente: el término fuente impacto da marea negra (oil spill effects [efectos del derrame de combustible]) se traduce como ecological catastrophe (catástrofe ecológica).

\section{Discusión}

En general, los resultados observados en la sección anterior confirman nuestra hipótesis sobre el uso de la variación terminológica en los textos especializados y las traducciones. Los resultados en la sección 4.1. confirman que la variación es un fenómeno frecuente en la comunicación especializada. El grado de variación y los tipos de variantes pueden cambiar según las preferencias o el estilo del autor, ya que el porcentaje de UC que tienen más de una variante difiere de un texto a otro, pero, en general, parece que la variación terminológica se emplea comúnmente por los expertos.

Con respecto al uso de la variación en las traducciones, los resultados cuantitativos de la sección 4.2. muestran que las UC que se caracterizan por la variación terminológica en los textos fuente también se caracterizan por la 
La variación terminológica en textos fuente y sus traducciones: un estudio piloto

variación en los textos meta. Para la mayoría de las UC en cada texto, se encontró el mismo número de variantes en los textos fuente y meta.

Los resultados del IVI (sección 4.3.) nos permiten confirmar nuestra hipótesis inicial de que, en general, las elecciones de términos motivadas cognitivamente en el texto fuente se reflejan en las traducciones. Sin embargo, en el texto «DC», la mayor proporción de unidades de traducción en las que la distancia cognitiva entre el término fuente y su traducción se marcaron como «1» $(22,97 \%)$ es notable en comparación con el texto «GN» $(4,12 \%)$ y el texto «SG» $(4,13 \%)$. De estos resultados, podemos deducir que en el texto «DC» se adoptó una estrategia de traducción mucho más libre, mientras que para los otros dos textos la traducción fue mucho más directa o literal. El nivel de conocimiento de los traductores profesionales con respecto al tema abordado en los textos fuente, su conocimiento de la lengua meta, su familiaridad con la terminología del campo temático, la disponibilidad de recursos terminológicos durante el proceso de traducción y la posible existencia de una política de traducción con respecto a la traducción de la terminología al inglés son algunos de los factores extralingüísticos que deben considerarse para tener en cuenta las diferencias entre el texto «DC» y los otros dos textos.

Por ejemplo, lo que sabemos sobre las traducciones en nuestro corpus es que los textos «SG» $\mathrm{y}$ «GN» Se tradujeron por traductores profesionales, mientras que la traducción del texto «DC» fue realizada por el autor del texto fuente, lo cual podría explicar por qué se adoptó una estrategia de traducción más libre en este último. Además, el hecho de que las traducciones de los textos «SG» « $\mathrm{GN} »$ aparecieran en las mismas actas de congreso de 2004 que los textos originales, puede explicar por qué los traductores profesionales aplicaron una estrategia de traducción más directa. La traducción del texto «DC» apareció en la revista International Journal of Oceans Affairs, dos años después de la publicación del texto fuente en las actas de congreso en 2007. En contraste con las actas de congreso que se dirigían principalmente a expertos del área, pescadores y empresarios, los lectores de la revista internacional son principalmente economistas marinos y expertos en ciencias marinas ${ }^{5}$, esto también puede explicar por qué se aplicó una estrategia de traducción más libre en el texto «DC» con respecto a la traducción de los términos fuente.

\section{Conclusión}

En este artículo se presentó una metodología que nos permitió examinar si la variación terminológica en los textos fuente especializados, que resulta de una elección terminológica motivada cognitivamente, se refleja en las traducciones y de qué manera. Un aspecto esencial en esta metodología es que los términos fuente que tienen un estado de referencia conjunta se agrupan y se consideran parte de la misma UC (sección 2.1.). La etiqueta que identifica cada UC o grupo de variantes terminológicas se usa para anotar los términos fuente y sus equivalentes en el corpus bilingüe. La anotación se realiza de forma semiautomática, esto significa que el programa que se desarrolló para esta tarea (sección 3.2.) primero solicita comentarios de un usuario antes de colocar automáticamente los términos fuente y las traducciones en las etiquetas de identificación correctas. Este programa se perfeccionará en el proyecto de investigación de CVC (sección 2.1.) para acelerar el análisis de la variación terminológica en futuros textos fuente y meta. 
La variación terminológica en textos fuente y sus traducciones: un estudio piloto

Se realizó un primer análisis cuantitativo con el objetivo de identificar las UC que se caracterizaban por presentar variación terminológica en los textos fuente. Al comparar el número de términos fuente y las traducciones para cada UC se observó que los traductores de los textos en nuestro estudio no siguieron la regla de consistencia presentada en la terminología prescriptiva. Sobre la base de los resultados de las distancias cognitivas entre los términos fuente y las traducciones y el índice de variación interlingüística para cada UC, pudimos concluir que las traducciones tienden a ser consistentes y reflejan lo más cerca posible las elecciones de términos de los textos fuente. El hecho de que en uno de los textos se aplicara una estrategia de traducción más libre en la traducción de ciertos términos fuente se explicó sobre la base de factores extralingüísticos relacionados con los procesos de traducción (sección 5.). Esto ilustra una vez más la importancia, en los estudios basados en corpus, de tener información disponible relacionada con la génesis de cada texto. Por un lado, es importante conocer más detalles sobre las personas involucradas en los procesos de escritura o traducción: por ejemplo, ¿cuál es su lengua nativa y qué tan familiarizados están con el tema y la terminología? Por otro lado, también es importante conocer más sobre la política de traducción, los recursos que se consultaron durante los procesos de escritura o traducción (por ejemplo, listas de terminología, documentos existentes, diccionarios generales o especializados) o las herramientas que se utilizaron (por ejemplo, memorias de traducción, herramientas de redacción técnica). Dicha información generalmente no se proporciona cuando se están compilando los corpus y a menudo es difícil de recopilar después de que el corpus se libera, sin embargo, esta información se consideró crucial en este estudio piloto para comprender las elecciones particulares con respecto a los términos fuente y las traducciones.

Los resultados que surgieron de este estudio también respaldan la idea de que, desde el punto de vista de la traducción, la noción de equivalencia terminológica puede diferir de la perspectiva onomasiológica tradicional según la cual la equivalencia terminológica se limita a variantes terminológicas (sinónimos o equivalentes de traducción) que denominan el mismo concepto. Los traductores aplican diferentes técnicas de traducción con miras a establecer la equivalencia entre un mensaje en la lengua fuente y su traducción a la lengua meta, lo cual a veces da como resultado unidades de traducción en las que el término fuente y su traducción reflejan diferentes conceptualizaciones. Un ejemplo de esto es el término gallego especies comerciais (commercial species [especies comerciales]) que se encontró traducido en el texto «DC» como affected species (especies afectadas), commercial stock (almacenamiento comercial) y market resources (recursos de mercado). Podría ser interesante examinar cómo los resultados de un estudio de variación terminológica en los textos fuente y meta podrían complementar aún más la información encontrada en los recursos de traducción especializada asistida por computador y de esta manera mejorar la calidad de dichos recursos. Creemos que este tema es un desafío interesante para futuras investigaciones basadas en corpus sobre la variación terminológica en la traducción especializada (próximamente, Kerremans).

Agradecimientos: Los autores desean agradecer a la profesora Rita Temmerman (CVC, Erasmus University College Brussels) por los comentarios realizados en un borrador anterior de este artículo. 


\section{Notas}

1. <http://cvc.ehb.be>, consultado el 13 de mayo de 2011.

2. <http://www.iula.upf.edu/iulaterm/tpresuk.htm>, consultado el 13 de mayo de 2011.

3. Puede encontrar más información sobre esta herramienta aquí: < http://neon.niederlandistik.fu-berlin.de/en/ textstat/>, consultado el 13 de mayo de 2011.

4. La Norma Reintegracionista afirma el origen común del gallego y el portugués y adopta algunas convenciones ortográficas de la lengua portuguesa. La Norma Oficial, creada en 1982 por la Real Academia de Lengua Gallega en el marco del Plan de Normalización Lingüística, sigue las convenciones ortográficas de la lengua española. 5. El público objetivo se indica explícitamente en la página de presentación de la revista, <http://www.elsevier. com/wps/find/journaldescription.cws_home/30453/description\#description> consultado el 13 de mayo de 2011.

\section{Referencias bibliográficas}

1. Bowker, L. (1997). You say «flatbed colour scanner», I say «colour flatbed scanner»: A descriptive study of the influence of multidimensionality on term formation and use with special reference to the subject field of optical scanning technology. Terminology, 4(2), 275-302.

2. Bowker, L. (1998). Variant terminology: frivolity or necessity? En 8th EURALEX-International Congress. Liege: University of Liege, 487-495.

3. Bowker, L. \& Hawkins, S. (2006). Variation in the organisation of medical terms: Exploring some motivations for term choice. Terminology, 12(1), 79-110.

4. Cabré, M. (1995). On diversity and terminology. Terminology, 2(1), 1-16.

5. Cabré, M. (2008). El principio de poliedricidad: la articulación de lo discursivo, lo cognitivo y lo lingüístico en Terminología (I). Ibérica, 16, 9-36.

6. Collet, T. (2004). Esquisse d'une nouvelle microstructure de dictionnaire spécialisé reflétant la variation en discours du terme syntagmatique. Meta, 49(2), 247-263.

7. Durieux, C. (1995). Texte, contexte, hypertexte. Cahier du CIEL (1994-1995), 214-228.

8. Felber, H. (1981). The Vienna School of Terminology, Fundamentals and Its Theory. Infoterm Series, 7, 69-86. 9. Fernández-Silva, S., Freixa, J., \& Cabré, M. (2009). Multiple Motivations in the Denomination of Concepts: The Case of «Production Area» in the Terminology of Aquaculture in French and Galician. Terminology Science and Research, 20. http://lipas.uwasa.fi/hut/svenska/iitf/tsr2005/vol20/

10. Freixa, J. (2002). La variació terminológica: Analisi de la variació denomitiva en textos de different grau d'especialització de varea de medi ambient. Barcelona: Institut Universitari de Lingüística Aplicada / Universitat Pompeu Fabra.

11. Freixa, J. (2006). Causes of Denominative Variation in Terminology: A Typology Proposal. Terminology, 
La variación terminológica en textos fuente y sus traducciones: un estudio piloto

12(1), 51-77.

12. Freixa, J., Fernández-Silva, S., \& Cabré, M. (2008). La multiplicité des chemins dénominatifs. Meta, 53(4), 731-747.

13. Gaudin, F. (2003). Socioterminologie : une approche sociolinguistique de la terminologie. Bruxelles : De Boek Duculot.

14. Guiraud, P. (1978). Les mots savants. Paris : Presses Universitaires de France.

15. Kageura, K. (2002). The Dynamics of Terminology: A Descriptive Theory of Term Formation and Terminological Growth. Amsterdam/Philadelphia: John Benjamins.

16. Kerremans, K. (2010). A Comparative Study of Terminological Variation in Specialised Translation. In: C. Heine \& J. Engberg (Eds.). Reconceptualizing LSP. Actas en línea del XVII Simposio Europeo de LSP 2009. Aarhus: Aarhus School of Business Aarhus University.

17. Kocourek, R. (1991). La langue française de la technique et de la science : vers une linguistique de la langue savante. Wiesbaden : Oscar Brandstetter.

18. Merkel, M. (1996). Consistency and Variation in Technical Translations. A Study of Translators' Attitudes. En Actas del Congreso de Traductología de la Unidad en Diversidad. Dublín. http://citeseerx.ist.psu.edu/viewdoc/ download?doi=10.1.1.44.2145\&rep=rep1\&type $=$ pdf

19. Myking, J. (2009). (Yet Another) Taxonomy of Motivations. Terminology Science and Research, 20. http:// lipas.uwasa.fi/hut/svenska/iitf/tsr2005/vol20/

20. Rogers, M. (2004). Multidimensionality in Concepts Systems: A Bilingual Textual Perspective. Terminology, $10(2), 215-240$.

21. Rogers, M. (2007). Lexical Chains in Technical Translation. A Case Study in Indeterminacy. In B. Antia (Ed.), Indeterminacy in Terminology and LSP. Amsterdam/Philadelphia: John Benjamins.

22. Sager, J. (1997). Term Formation. In S. E. Wright \& G. Budín (Eds.). Handbook of Terminology Management (pp. 25-41). Amsterdam/Philadelphia: John Benjamins.

23. Suárez de la Torre, M. (2004). Análisis contrastivo de la variación denominativa en textos especializados: del texto original al texto meta. Barcelona: Institut Universitari de Lingüística Aplicada/Universitat Pompeu Fabra.

24. Temmerman, R. (2000). Towards New Ways of Terminology Description: The Sociocognitive Approach. Amsterdam/Philadelphia: John Benjamins.

25. Toury, G. (1995). Descriptive Translation Studies and Beyond. Amsterdam/Philadelphia: John Benjamins.

26. Wüster, E. (1979/1991). Einführung in die Allgemeine Terminologielehre und Terminologische Lexikographie.

En L. Bauer (Ed.), Schriftenreihe der technischen Universitat Wien. Wien/NY: Springer. 
La variación terminológica en textos fuente y sus traducciones: un estudio piloto

\section{Anexo 1: Referencias de los corpus}

\section{Textos en gallego:}

(SG) Suris-Regueiro, J. C. \& Garza-Gil, D. (2004). Avaliación de danos directos e indirectos. Metodoloxía e plan de traballo para o caso do Prestige. En A. Prada Blanco \& M. X. Vázquez Rodríguez (Eds.), Efectos económicos, sociais e ambientais da marea negra do Prestige (pp. 311-351). Madrid: Consello da Cultura Galega.

(GN) García Negro, M. (2004): Consideración sobre o estudo dos danos económicos nos sectores productivos directamente vinculados ás actividades mariñas. O caso do Aegean Sea. En A. Prada Blanco \& M. X. Vázquez Rodríguez (Eds.), Efectos económicos, sociais e ambientais da marea negra do Prestige (pp. 354-419). Madrid: Consello da Cultura Galega.

(DC) Villasante, C. S., García Negro, M. \& Carballo Penela, A. et al. (2007). Sobre a valoración económica dos efectos do vertido do Prestige na pesca comercial na Costa da Morte. En X Foro dos Recursos Mariños e da Acuicultura das Rías Galegas e I Foro Iberoamericano dos Recursos Mariños e da Acuicultura, 665-682.

\section{Textos en inglés:}

Suris-Regueiro, J. \& Garza-Gil, D. (2004). Evaluation of Direct and Indirect Damages. Methodology and Work Program for the Prestige Case. En A. Prada Blanco \& M. X. Vázquez Rodríguez (Eds.), Economic, Social and Environmental Effects of the Prestige Spill (pp. 311-351). Madrid: Consello da Cultura Galega.

García Negro, M. (2004). Considerations on the Study of Economic Damage in Productive Sectors Directly Linked with Marine Activities. The Case of the Aegean Sea. En A. Prada Blanco \& M. X. Vázquez Rodríguez (Eds.), Economic, Social and Environmental Effects of the Prestige Spill (pp. 354-419). Madrid: Consello da Cultura Galega.

García Negro, M., Villasante, C. S., Carballo Penela, A., et al. (2009). Estimating the Economic Impact of the Prestige Oil Spill on the Death Coast (NW Spain) Fisheries. Marine Policy. The International Journal of Oceans Affairs, 33, 8-23. 